\title{
Retention of an appropriate astrocytic response to retinoids by human brain tumors composed of predominantly glial fibrillary acidic protein-positive cells
}

\section{Elizabeth Noll, and Peter McL. Black, M.D., Ph.D.}

Division of Neurosurgery and Brain Tumor Laboratories, Brigham \& Women's Hospital, Boston, Massachusetts 02115

It has been demonstrated that glial fibrillary acidic protein (GFAP)-positive astrocyte precursors will differentiate in response to application of retinoic acid (RA), whereas GFAP/oligodendrocyte type 2-astrocyte progenitors will be inhibited from differentiating and continue to be mitotically active in the presence of RA. The authors sought to determine if cells taken from glial tumors that were GFAP positive retained the ability to differentiate following application of RA in vitro, as their normal astrocytic counterparts do. Primary cultures of seven astrocytic tumors were observed to have significantly fewer cells following 1 month of continuous exposure to $100 \mu \mathrm{M}$ RA. Comparisons with sister control cultures indicated that in control conditions the tumor cells had undergone proliferation, whereas the number of cells in the RA-exposed cultures remained closer to the number of cells initially plated. This response to RA was demonstrated to be specific to the astrocytic tumors by virtue of the fact that cultures of normal brain and an anaplastic ependymoma both showed a strong proliferative response to retinoids.

\section{Key Words * glioma * glial fibrillary acid protein * astrocytic response}

During embryonic central nervous system (CNS) development, intracellular signaling has been demonstrated to be critical for the regulation of cell division and the timing of precursor cell differentiation. A wealth of data suggest that a carefully balanced orchestration of mitogenic, differentiative, and apoptotic stimuli leads to the normal development of a functional CNS containing the full complement of cells and cell types. To generate the diversity of cells required in the vertebrate CNS, multipotent progenitor cells reproduce and become sequentially more restricted in their ability to produce different cell types as development progresses.[13,14] It is now clear that some progenitor cells perdure in the adult nervous system,[20] presumably to repair minor insults and to provide general maintenance. These precursor cells can and do divide to maintain the necessary number of cells to support the CNS throughout a lifetime.

The persistence into postnatal life of these glial precursor cells has far reaching implications for the genesis of brain tumors, because we know that where there is cell division, there is potential for 
neoplastic transformation. The legitimacy of using developmental biology as a framework to find means of controlling glial tumorigenesis has been supported by several studies in which parallels between the antigenic expression profile or cellular behavior of cell lines that originated from human brain tumors and their normal CNS counterparts have been reported.[5,7,12,24,25] One criticism of these studies has been that because they were performed on cell lines that underwent numerous passages in vitro prior to examination of their antigenic expression or response to stimuli, it is unclear how closely the cells examined resembled the cells that were initially found in vivo. Furthermore, most investigators in these studies examined predominantly the antigenic phenotype or the response to defined stimuli, but made only limited efforts to correlate antigenic expression with a specific response to defined stimulus. Therefore, it is difficult to surmise from the limited number of cell lines examined in these studies how well the antigenic phenotype of neoplastic cells derived from human brain tumors correlates with the normal response of their nonneoplastic counterparts.

One of the most widely examined stimuli in human gliomas is retinoic acid (RA). Retinoic acid has been demonstrated to drive normal, immature astrocytes to differentiate and express high levels of the astrocyte-specific marker glial fibrillary acidic protein (GFAP)[2] during perinatal development.[29] Notably, RA has precisely the opposite effect on oligodendrocyte precursor cells, which do not express GFAP and which are inhibited from differentiating in the presence of RA.[10,19]

The oligodendrocyte type 2-astrocyte $(\mathrm{O} 2 \mathrm{~A})$ progenitor, first isolated from the rat optic nerve, $[15,27]$ is a bipotential glial progenitor that gives rise to oligodendrocytes around the time of birth and a population of type-2 astrocytes shortly thereafter.[15,23] This glial progenitor is antigenically distinguishable from purely astrocytic progenitors, including the failure to express GFAP.[6,15,22] The antigenic phenotype, proliferative and migratory capacity, as well as the ability to give rise to at least two distinct cell types, are characteristics that seem to be common to O-2A progenitors found throughout the perinatal and adult CNS.[1,8,11,18,28]

One can distinguish glial cells that are astrocytic from oligodendroglial precursors by assessment of GFAP expression and permanent cessation of cell division (a differentiated response) in the presence of RA. If the cells express GFAP prior to exposure to RA and cease to divide on introduction of RA, then it can be said that the cell is, or behaves like, an astrocyte. However, if a glial cell does not express GFAP and does not cease to divide on introduction of RA, then it can be said that it is not astrocytic based on its antigenic expression and response to the stimulus.

Are these antigenic and behavioral characteristics of normal glia at all retained by neoplastic glia? This is the question we sought to examine in this study. It is entirely possible that one of the reasons that neoplastic astrocytes divide inappropriately is that they have lost the ability to respond to RA, due to aquisition of somatic mutations within the receptor(s) or downstream signal transduction machinery required to mediate differentiative signals. Similarly, it could be that glioma cells have acquired constitutively activating mutations in signal transduction pathways that mediate the effects of mitosis-inducing signals. If this were the case, then one might expect that any input from differentiative signals would be drowned out, as it were, by the constitutive signal to divide. We chose to address these possibilities directly, by examining the effect of RA on primary cultures established from human gliomas.

\section{MATERIAL AND METHODS}

Tissue used in this study was obtained following the protocols submitted and approved by the respective 
Human Research Committees of Brigham and Women's Hospital and The Children's Hospital of Boston, and in accordance with federal, state, and institutional guidelines.

After resection of a tumor, a sample of tissue is designated "for special studies" by the attending neurosurgeon. After determining that an accurate diagnosis can be made by examining the samples already in the possession of the neuropathology department, a gross analysis of the entire specimen designated for special studies is performed and recorded, and then the sample is divided into approximately equal portions by the attending neuropathologist. Using sterile technique, the neuropathologist makes sure that each of the two portions contain representative proportions of the tumor. Following release of half of the tissue by the neuropathologist, the specimen is immediately taken to the lab to be processed for tissue culture. The portion of each specimen designated for special studies that is retained by the neuropathology department is examined and the description and diagnosis for the tissue corresponding to that placed in culture is noted in the pathology report, along with all other specimens. The "normal" brain cells were obtained from a 3-year-old boy who underwent a left hemispherectomy for Rasmussen's disorder. This tissue was described as nonneoplastic brain tissue by the attending neuropathologist. The ependymal tumor was described as an anaplastic ependymoma.

\section{In Vitro Analysis}

Using sterile technique, tumor tissue is dissociated and placed in culture by using a slight modification of the method described by Noll and Miller.[19] Briefly, the tumor is finely minced and suspended in trypsin-ethylenediaminetetraacetic acid (EDTA) solution (T-555; Sigma Chemical Co., St. Louis, MO) and then incubated at $37 \mathrm{~s} \mathrm{C}$ for between 10 and 30 minutes depending on how finely minced the tissue is (cells already in culture are dissociated in the flask for 5-10 minutes). Following trypsin digestion, an equal volume of medium containing $10 \%$ fetal bovine serum (FBS) is added to the tube to inactivate the trypsin. Cells are then pelleted for 10 to 15 minutes at 3500 to $4000 \mathrm{rpm}$ and resuspended in medium. Cells are then plated at medium to high density $\left(10^{5}-10^{6}\right.$, depending on the size of the sample received) on 12-mm round glass coverslips (63-3009; Carolina Biological, Burlington, NC) that have been cleaned ( 24 hours in $2 \mathrm{~N} \mathrm{HCl}$, followed by 24 hours in running $\mathrm{dH}_{2} \mathrm{O}$ ), sterilized in an autoclave, and placed in 24-well polystyrene tissue culture plates. Prior to plating cells, the coverslips are treated with sterile poly-L-lysine solution (in $\mathrm{dH}_{2} \mathrm{O}$ ) for 30 to 60 minutes at $37 \mathrm{~s} C$, rinsed with $\mathrm{dH}_{2} 0$, and allowed to dry completely. Cells are suspended in medium to permit an appropriate number to be plated in a volume of $75 \mu \mathrm{l} /$ coverslip and then incubated at $37 \mathrm{~s} \mathrm{C}$ with $5 \% \mathrm{CO}_{2}$. Cells are allowed to attach to the coverslips for 1 to 3 hours, and then the wells are gently flooded with approximately $1 \mathrm{ml}$ of medium. Cultures are left undisturbed for at least 24 hours to permit cells to attach firmly to the coverslips and to fully recover from trypsin exposure.

For the analysis of the effects of RA on growth, $3 \mu \mathrm{l}$ of $1 \mathrm{mM}$ all-trans RA (R-2625; Sigma Chemical Co.) suspended in $100 \%$ ethanol (ETOH) was added to each experimental culture $(1 \mathrm{ml}$ of media/well for a final concentration of $3 \mu \mathrm{M} \mathrm{RA}$ ) every 21 to 24 hours for the duration of the experiment. After 1 month of daily RA application, cultures were assayed for growth by staining with Hoechst (after fixation) and GFAP (see Immunohistochemical Analysis) followed by counting the number of nuclei in 10 consecutive fields at a magnification of X 40 .

\section{COMPOSITION OF MEDIUM}

The medium used in this study was modified from the N2 serum-free growth medium originally 
described by Bottenstein and Sato.[3] Several reports of modifications to this medium are found in the literature,[28,19] and it has been demonstrated that glial cells (including oligodendrocytes) from rat can be maintained indefinitely in vitro in these conditions. It has also been demonstrated that oligodendrocytes from fetal rat spinal cord will differentiate according to the proper in vivo schedule when plated at high density and maintained in medium containing the proper supplements.[26,28] The $\mathrm{N} 2$ medium used in the present study has been further modified to support human glial cells and was observed to support normal growth and development of astrocytes and oligodendrocytes obtained from human fetal spinal cord (Noll and Black, unpublished observations) indicating the medium contains the neccessary components to mimmick the in vivo situation closely.

The following stock solutions are prepared, aliquoted and stored at -20 šC until use: insulin/transferrin/selenium (ITS) supplement (I-1884; Sigma) suspended in Dulbecco's modified Eagles Medium (DMEM) [1 vial ITS thoroughly suspended in $25 \mathrm{ml}$; human progesterone (P-6149; Sigma) suspended in 100\% ETOH [1 mg/10 ml]; putrescine (P6024; Sigma) suspended in DMEM [10 mg/10 $\mathrm{ml}$; 3,3',5 triiodo-L-thyronine (T-6397; Sigma) suspended in 100\% ETOH [10 mg/10 ml]; thyroxine (T-0379; Sigma) suspended in 100\% ETOH [2 mg/2 ml]; fraction V bovine serum albumin (BSA) (1\% suspended in DMEM; A-4503; Sigma); heat-inactivated FBS (30 minutes at 56 šC; A-1115-L; Hyclone Laboratories, Inc., Logan, UT); and penicillin/streptomycin solution (Gibco/BRL, Rockville, MD).

To make supplemented medium, we added the following amounts of stock solutions described above to $500 \mathrm{ml}$ of high glucose DMEM (11965-068; Gibco/BRL) followed by filter sterilization with a 0.2- $\mu \mathrm{m}$ filter: $2.5 \mathrm{ml}$ ITS stock solution, $5 \mu \mathrm{l}$ progesterone stock solution, $800 \mu \mathrm{l}$ putrescine stock solution, 16.5 $\mu \mathrm{l}$ triiodo-L-thyronine stock solution, $20 \mu \mathrm{l}$ thyroxine stock solution, $5 \mathrm{ml}$ fraction V BSA stock solution, and $50 \mathrm{ml}$ heat-inactivated FBS. We stored the medium in the dark at 4 šC and used it within 10 to 14 days. We added $1 \mathrm{ml}$ of medium per well to a 24 -well plate ( $3 \mathrm{ml} / \mathrm{T} 25$ flask; $25 \mathrm{ml} / \mathrm{T} 75$ flask). While growing in culture, medium was changed once every 7 days.

\section{Immunohistochemical Analysis}

Coverslips are removed from 24-well plates and fixed in 4\% methanol-free formaldehyde (18814; Polyscience, Niles, IL) solution made in phosphate-buffered saline (PBS) (P-4417; Sigma) for 10 minutes at room temperature. Slides are then rinsed three times in PBS, followed by a 20-minute incubation in 2\% FBS in PBS. As a control, human fetal spinal cord cultures established from 19 -week-old fetuses are processed and stained in parallel.

The GFAP antibody (Neural Cell Typing Set, 1444 638; Boehringer Mannheim) was diluted 1:10 in a solution of 1:1 PBS and FBS. Incubation in primary antibody was performed in a moist chamber for 1 to 2 hours at room temperature. The antibody solution is then drained off and slides are rinsed four times in PBS at room temperature. A 1:20 dilution of sheep anti-mouse immunoglobulin fluorescein isothiocyanate-conjugated antibody ( 821 462; Boehringer Mannheim) is then applied to the coverslips, which are incubated for 30 minutes at room temperature in a moist, darkened chamber. (It should be noted that all other anti-mouse secondary antibodies we tried [all made in goat] cross-reacted with a subpopulation of what appeared to be astrocytes, both in tumor samples and in fetal spinal cord sections.) The antibody solution is then drained off and slides are washed extensively (four-six washes in PBS at room temperature), and mounted in $80 \%$ glycerol/SlowFade solution (Molecular Probes, Inc., Eugene, OR). For Hoechst staining following incubations for GFAP staining, a 1:1000 Hoechst solution in PBS is prepared and sections are incubated for 8 minutes at room temperature, and then rinsed extensively 
(four-six washes in PBS). The slides are visualized using an Axiophot microscope (Carl Zeiss, Thornwood, NY) with an ultraviolet light source. The mean number of cells per field (+/- the standard deviation) is determined by counting 10 consecutive $\mathrm{X} 40$ fields from three separate cultures grown in either experimental or control conditions for each specimen.

\section{RESULTS}

Using standard methods that permit long-term survival of glial cells in vitro (see Materials and Methods), five glioblastomas multiforme and two astrocytomas (one fibrillary, one anaplastic) were placed in culture immediately following resection. After allowing for a period of undisturbed growth, cells from each tumor were replated and randomly designated as control or RA-treated cultures. After attachment, the cultures were either provided with fresh media alone (control) or fresh media with $3 \mu \mathrm{M}$ RA. Following a 3-week period of exposure to $3 \mu \mathrm{M}$ RA, both the control and RA-treated cultures were examined for growth.

To permit a comparison of the effects of the RA exposure on a substantial number of tumors, we chose to compare the average number of cells in the RA-exposed cultures as a percentage of the average number of cells in the corresponding control cultures of that particular tumor. This is because the number of cells we are able to place in culture is directly related to the size of the sample we receive, which is usually indicative of the size of the tumor at the time of resection. In many cases, the size of tumor at the time of resection is relatively small, because small tumors in certain areas of the brain can induce significant neurological symptoms. Therefore, if we were to decide to plate cells from different tumors at precisely the same density, we would necessarily be preselecting the tumors examined based on their size at the time of resection. As this might bias our results, we chose to plate tumor cells at high density $\left(10^{6}\right)$ whenever our sample size permitted us to do so, with cells taken from the original plating or first passage whenever possible (or up to the third passage in the case of extremely small samples). Studies of glial development indicate that the timing and relative number of glial cell types that differentiate in vitro are comparable to those observed in vivo when the cells are plated at a density of $10^{6}$ in defined culture conditions. $[18,22,28]$ However, when the tumor sample size was too small to permit plating cells from a low passage number at high density, the cells were plated at a lower density (not less than $10^{4}$ ). In cases such as this, one would not be able to compare the difference in absolute number of cells between tumors plated at lower densities with those plated at higher densities, making the absolute number of cells in control compared with RA-exposed cultures uninformative relative to other tumors.

Because cells in the adult brain never exist at low density in vivo, we used the highest density of cells possible while maintaining the lowest possible number of passages, as each passage is likely to impose an undesirably heavy selection on the cells used in these experiments. This approach, however, requires that to compare the effects of RA exposure between tumors, the number of cells found in each of the control cultures must be "normalized" to $100 \%$, and the number of cells in the RA-treated cultures must be expressed as a percentage of its control. It should be noted that the number of cells plated per coverslip was recorded for each tumor, and in all cases there were more cells in the control cultures 21 days after the experiment commenced than were originally plated (ranging from two-10 times the number of cells originally plated). This analysis demonstrated that all of the tumors tested were inhibited, to varying degrees, from growing in the presence of RA (Fig. 1). 


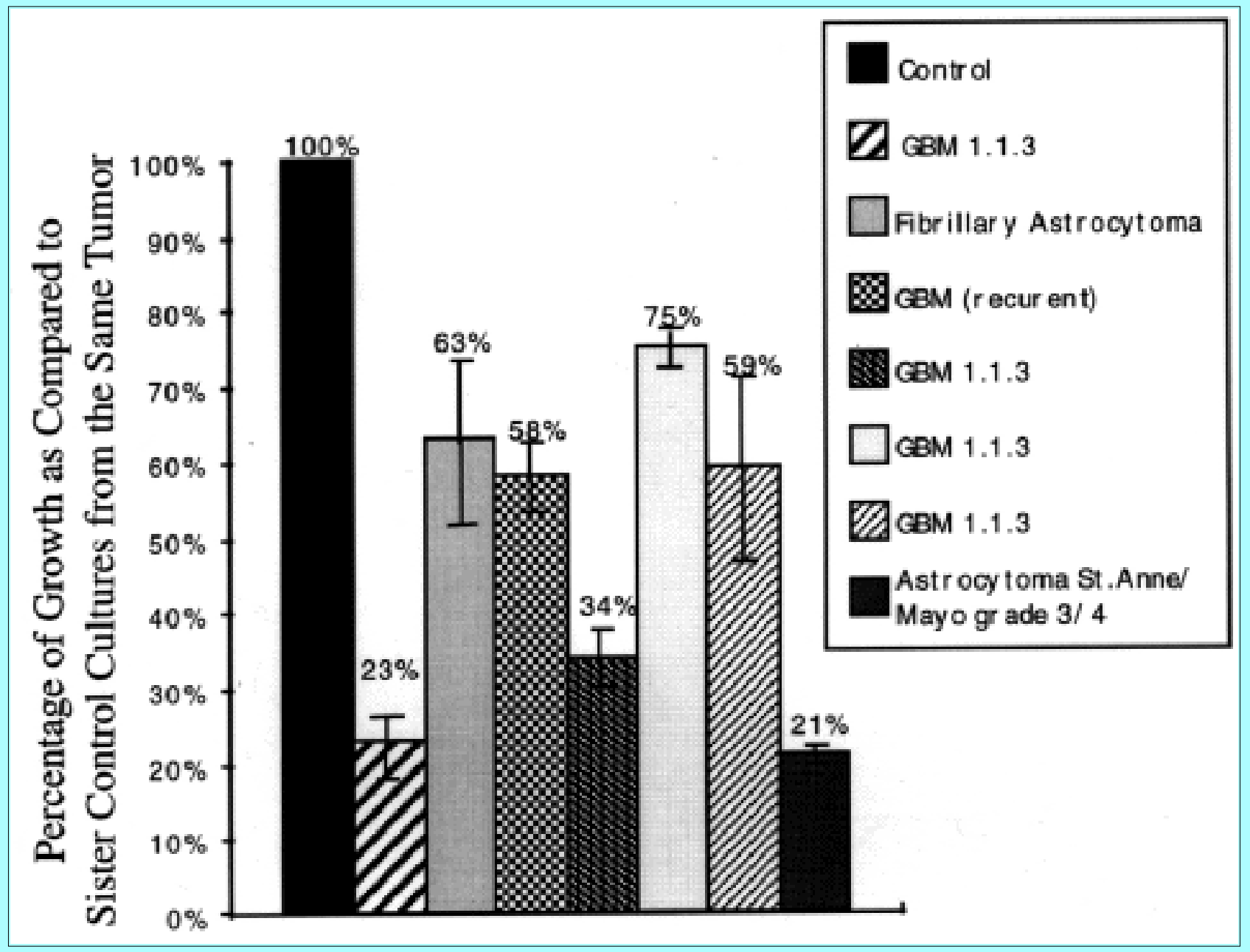

Fig. 1. Graph showing that following 1 month of continuous exposure to RA, all GFAP positive astrocytic tumors showed reduced growth compared with each individual tumor's matched control cultures, which were taken to represent $100 \%$ of expected growth.

There are several things worth noting about these data. Although the reduction in growth observed in the RA-exposed cultures varied, the nature of the response observed in all tumors was similar (inhibition of growth). In no case was the response of these astrocytic tumors to RA exposure to increase the amount of cell division compared to the unexposed controls. Similarly, in no case were the astrocytic cells observed to disregard the differentiative influence of RA completely. In all cases cells derived from these GFAP positive astrocytic tumors were observed to respond with a reduction in growth compared with their unexposed sister cultures; a response that has been demonstrated to be an identifying characteristic of normal GFAP positive astrocytes

While the nature of the in vitro response of astrocytic tumors was similar, the strength of the response fell into one of two general classes: those tumors that had a moderate reduction in growth in response to RA (between $21 \%$ and 35\%) and those that had a large reduction in growth in response to RA (between $58 \%$ and $75 \%$ ). This difference in the response to RA was not observed to correspond to differences in the density of cells at the time the experiment was undertaken and it was not observed to correspond to the pathological gradation of the tumors.

All the astrocytic tumors we examined exhibited a qualitatively similar response to RA exposure (inhibition of growth), but the specificity of this response needed to be demonstrated. The specificity of the inhibition of growth of astrocytic tumors was determined by examining the effects of RA exposure on 
"normal" brain, and on nonastrocytic tumors such as ependymomas. When RA was applied to "normal" brain cells obtained from a 3-year-old boy who underwent a hemispherectomy for Rasmussen's syndrome there was a moderate increase in the number of cells in the RA-exposed cultures compared with their control cultures (Fig. 2).

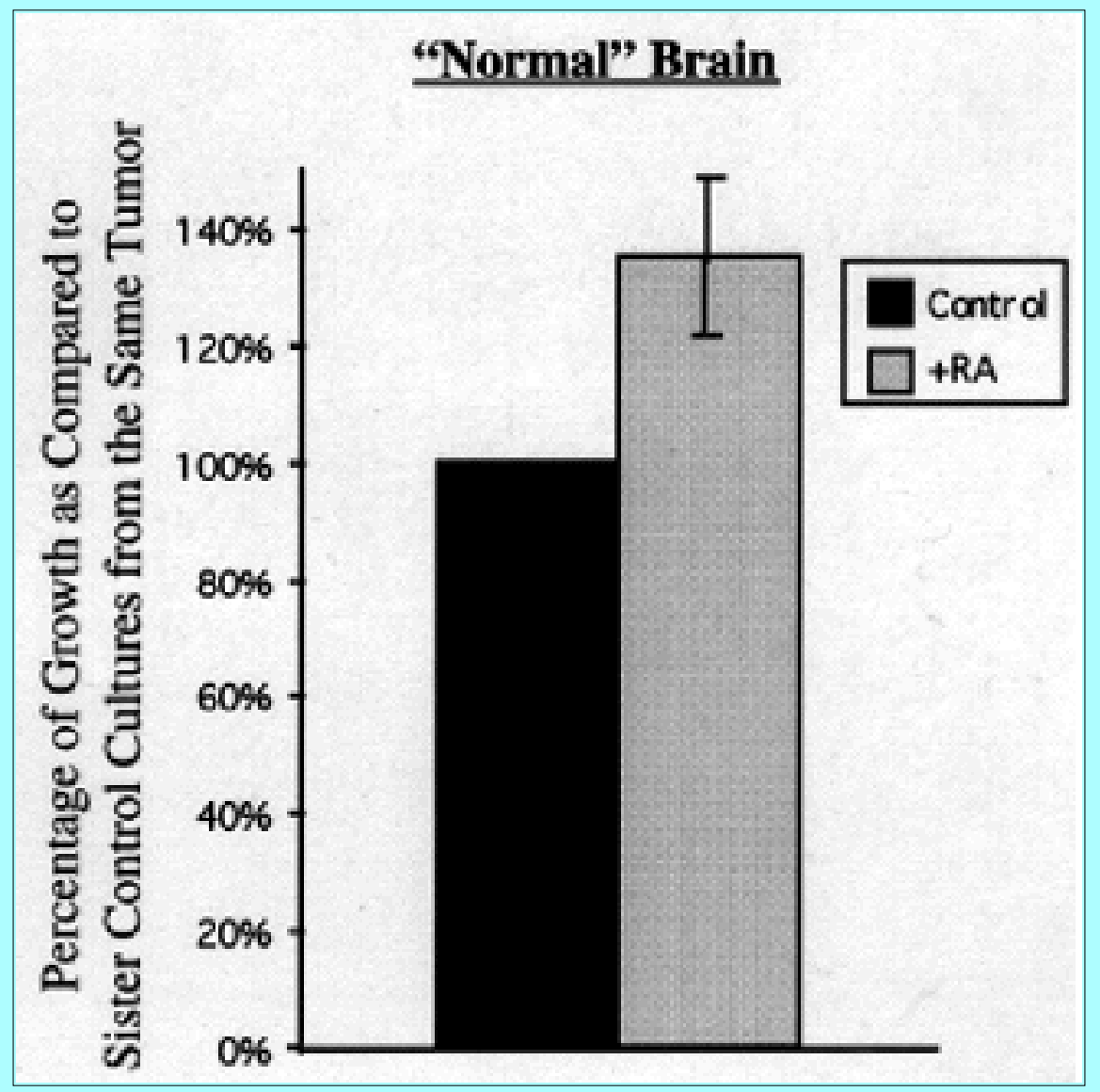

Fig. 2. Graph showing that when exposed to $100 \mu \mathrm{M}$ RA for a period of 1 month, there was a proliferative response in cultures of normal brain that contained a variety of cell types.

In the case of cells obtained from the resection of an anaplastic ependymoma, there was a dramatic increase in cell number in cultures exposed to RA compared their control cultures (Fig. 3). These data support the hypothesis that the nature of the response of astrocytic tumors to RA exposure (growth inhibition) in the previous set of experiments reflects the biological response of the astrocytic cells that compose the tumor. 


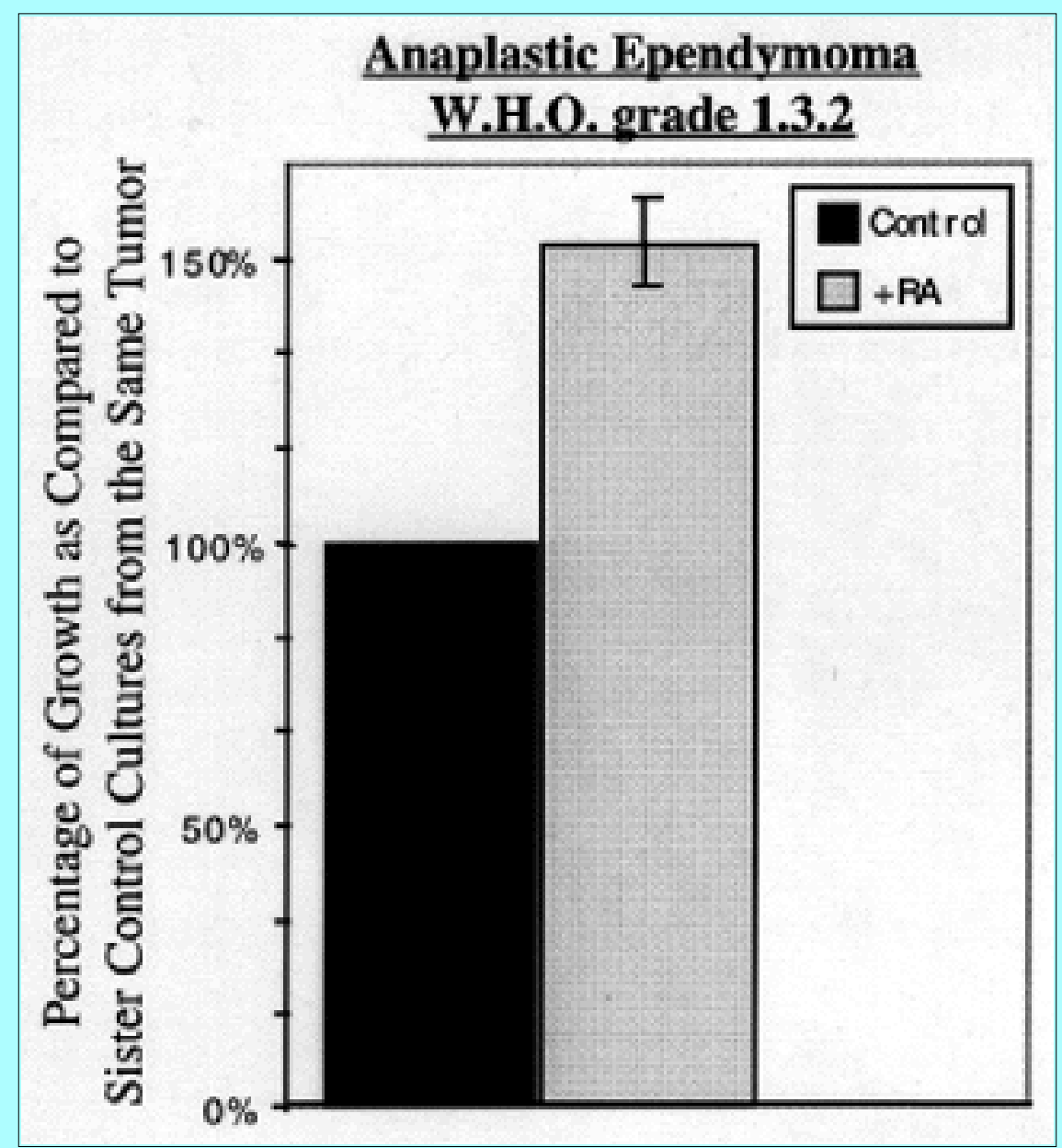

Fig. 3. Graph showing that when exposed to $100 \mu \mathrm{M}$ RA for a period of 1 month, there was a marked proliferative response of ependymoma cells (> 50\% over control). Note that this is an opposite response to RA as compared with the GFAP positive astrocytic tumors.

\section{DISCUSSION}

Our experiments were designed to determine if we could consistently observe an effect on the behavior of cells from primary brain tumors said to be GFAP positive, which would mimick the effect of RA on normal GFAP-positive astrocytes and to determine the specificity of the response to RA. Using the original plating or the first or second passage of tumors containing GFAP-positive cells (as determined ex vivo in section), we were able to demonstrate that RA inhibits continued expansion of tumor cells. Because all of the astrocytic tumors we examined showed a significant reduction in growth when exposed to RA and all of these cultures contained GFAP-positive cells, we can conclude that cells within astrocytic tumors behave in a manner consistent with that of their nonneoplastic astrocytic counterparts. Therefore, the expression of GFAP, even in neoplastic cells, can be correlated with an astrocyte-like response to RA, suggesting that the receptor(s) and signal transduction machinery required for RA to ellicit a differentiative response in normal astrocytes, remains intact in at least some subpopulation of astrocytic tumors.

We next sought to determine the specificity of this response by examining the effects of RA on nonneoplastic human brain, and a non-astrocytic tumor. In response to RA exposure, cells from either non-neoplastic human brain or an anaplastic ependymoma underwent increased cell division relative to their matched controls: a response completely opposite from that of all the GFAP-positive astrocytic tumors. This experiment demonstrates that the inhibited proliferation observed in GFAP-positive tumors 
does not simply reflect a broad, nonspecific inhibition of proliferation in all CNS cells. To the contrary, there was nearly a 50\% increase in the number of cells in cultures of ependymoma cells that were exposed to RA.

It is interesting to note that the "normal brain" cell underwent increased cell division in response to RA. On first glance this result may appear somewhat surprising because the vast majority of glial cells in the postnatal brain are thought to be astrocytic in origin. There are two points that are likely to be directly relevant to our observations. First, the patient from whom the normal cells used in this experiment were isolated was a 3-year-old child. Oligodendrocyte type 2-astrocyte progenitors, which are inhibited from differentiating and will continue to divide at a brisk pace in the presence RA, $[10,19]$ are still present in the mature brain and would be expected to be found in significant numbers in a young child, as the majority of gliogenesis in vertebrates occurs postnatally. Second, the fact that ependymomas are predominantly a tumor found in the pediatric population suggests that ependymal precursor cells disposed to mitotic activity are present in young children. Our observation that RA exerts a positive effect on cell proliferation in cells isolated from an ependymoma suggests that RA may serve as a mitogen for ependymal cells. Clearly, further experiments will be required to demonstrate this definitively.

Rather than finding that the inhibitory effects of RA on the tumors containing GFAP-positive cells fell along a broad continuum, we discovered that the reduction in cell proliferation fell into one of two distinct categories: those tumors that were moderately inhibited from expanding (58-75\% of expected growth) and those that were strongly inhibited from expanding (21-34\% of expected growth). Although this was something of a surprise to us at first, it might have been predicted.

It is clear that there are at least two distinct types of astrocytes, Type 1 and Type 2.[16,17] The Type 1 astrocyte appears to arise from a separate precursor cell from the Type 2 astrocyte, which arises from the bipotential O2A progenitor.[14] As discussed previously, the uncommited O2A progenitor is completely inhibited from differentiating in the presence of RA and will continue to divide as long as there is a sufficient amount of platelet-derived growth factor present. Oligodendrocyte type 2-astrocyte progenitors that are induced to become astrocytes by cilliary neurotrophic factor, and that have become GFAP positive will be driven to differentiate rapidly by RA. Oligodendrocyte type 2-astrocyte progenitors commited to become oligodendrocytes, having become $\mathrm{O} 4$ positive,[19] will completely unresponsive to RA and will progress through the stages of differention as they would in the absence of RA.

It is possible that the tumors moderately inhibited from continued growth represent a population of neoplastic O2A progenitor cells. This hypothesis would predict that if tumors contained neoplastic O2A cells, only those cells that had begun to express GFAP at the time of RA exposure, and therefore said to have been commited to an astrocytic fate, would be inhibited from any further division and differentiate. In support of this idea, we found that there was a subpopulation of strongly GFAP-positive cells in the RA-exposed cultures, which were interspersed with substantial numbers of GFAP-negative cells (Noll and Black, unpublished observation). If this hypothesis is correct, it follows then that the tumors that were strongly inhibited from further division were mostly composed of cell commited to an astrocytic fate, and therefore most of the cells responded to RA by cessation of proliferation. Although this is clearly an untested hypothesis, it is nonetheless a very intriguing possibility worth pursuing.

We thought that it was important to determine whether the dramatic growth arrest achieved in the presence of exogenous RA was a transient phenomenon or if this growth arrest would continue even 
when exogenous RA was no longer provided. Notably, when the numbers of cells in these previously RA-treated cultures were determined a full month after withdrawing RA treatment there was a very limited increase in cell number $(<15 \%$ in all cases; data not shown), which is consistent with the previous RA exposure inducing the differentiation of the neoplastic astrocytic component of the tumor. In support of this conclusion, when GFAP expression was assayed in these cultures, it was noted that the cells expressed very high levels of GFAP.

Because oral administration of RA is quite safe and ingested RA crosses the blood-brain barrier quite readily, it could be directly determined whether the inhibiton of astrocytic growth observed in vitro would also occur in vivo in patients with predominantly GFAP-positive tumors. Indeed, some studies have been performed in which the therapuetic effects of oral administration of RA for gliomas have been examined. These studies have yielded disappointing results thus far.[4,9,21] However, we would suggest that to identify patients who could derive significant benefit from RA treatment and therefore test the hypothesis we have outlined, one would have to test individual resected tumors directly in vitro. Patients with so-called "mixed gliomas" would perhaps be expected to show a weak or moderate response to RA, because by definition more than one glial cell type is likely to be present in their tumors. By analyzing the results of a clinical trial in which the nebulous diagnoses of glioma, or mixed glioma were made, one could completely mask any indication of the utility of RA in patients with a specific subset of predominantly astrocytic tumors.

Indeed, the observation that the response of cells contained in GFAP-positive tumors fell into two distinct classes points to the necessity of testing more than one tumor (or tumor cell line) to identify subtle, yet perhaps critical differences among tumors that have been histologically classified as being related, if not identical. At this point, one thing appears quite clear: if we hope to identify novel, effective therapies for brain tumors, we will need to design and perform studies that take into account the wide variety of cell types that exist in the brain, the biology of each cell type, and each cell type's distinctly different response to identical stimuli. Further, these studies will have to be of a substantial enough scale that subtle differences among tumors can be detected.

\section{References}

1. Behar T, McMorris FA, Novotny EA, et al: Growth and differentiation properties of O-2A progenitors purified from rat cerebellar hemispheres. J Neurosci Res 21:168-180, 1988

2. Bignami A, Eng LF, Dahl D, et al: Localization of the glial fibrillary acidic protein in astrocytes by immunofluorescence. Brain Res 43:429-435, 1972

3. Bottenstein JE, Sato GH: Growth of a rat neuroblastoma cell line in serum-free supplemented medium. Proc Natl Acad Sci USA 76:514-517, 1979

4. Defer GL, Adle-Biassette H, Ricolfi F, et al: All-trans retinoic acid in relapsing malignant gliomas: clinical and radiological stabilization associated with the appearance of intratumoral calcifications. $\mathbf{J}$ Neurooncol 34:169-177, 1997

5. Dirks PB, Patel K, Hubbard SL, et al: Retinoic acid and the cyclin dependent kinase inhibitors synergistically alter proliferation and morphology of U343 astrocytoma cells. Oncogene 15:2037-2048, 1997 
6. Eisenbarth GS, Walsh FS, Nirenberg M: Monoclonal antibody to a plasma membrane antigen of neurons. Proc Natl Acad Sci USA 76:4913-4917, 1979

7. Gillaspy GE, Miller RH, Samols D, et al: Antigenic and differentiative heterogeneity among human glioblastomas. Cancer Lett 68:215-224, 1993

8. Ingraham CA, McCarthy KD: Plasticity of process-bearing glial cell cultures from neonatal rat cerebral cortical tissue. J Neurosci 9:63-69, 1989

9. Kaba SE, Kyritsis AP, Conrad C, et al: The treatment of recurrent cerebral gliomas with all-trans-retinoic acid (tretinoin). J Neurooncol 34:145-151, 1997

10. Laeng P, Decimo D, Pettmann B, et al: Retinoic acid regulates the development of oligodendrocyte precursor cells in vitro. J Neurosci Res 39:613-633, 1994

11. Levi G, Gallo V, Ciotti MT: Bipotential precursors of putative fibrous astrocytes and oligodendrocytes in rat cerebellar cultures express distinct surface features and neuron-like aminobutyric acid transport. Proc Natl Acad Sci USA 83:1504-1508, 1986

12. Maria BL, Steck PA, Yung WK, et al: The modulation of astrocytic differentiation in cells derived from a medulloblastoma surgical specimen. J Neurooncol 7:329-338, 1989

13. Mayer-Proschel M, Rao MS, Noble M: Progenitor cells of the central nervous system: a boon for clinical neuroscience. J NIH Res 9:31-36, 1997

14. McKay R: Stem cells in the central nervous system. Science 276:66-71, 1997

15. Miller RH, David S, Patel R, et al: A quantitative immunohistochemical study of macroglial cell development in the rat optic nerve: in vivo evidence for two distinct glial cell lineages. Dev Biol 111:35-41, 1985

16. Miller RH, Ffrench-Constant C, Raff MC: The macroglial cells of the rat optic nerve. Annu Rev Neurosci 12:517-534, 1989

17. Miller RH, Szigeti V: Clonal analysis of astrocyte diversity in neonatal rat spinal cord cultures. Development 113:353-362, 1991

18. Noll E, Miller, RH: Oligodendrocyte precursors originate at the ventral ventricular zone, dorsal to the ventral midline region in the embryonic rat spinal cord. Development 118:563-573, 1993

19. Noll E, Miller, RH: Regulation of oligodendrocyte differentiation: a role for retinoic acid in the spinal cord. Development 120:649-660, 1994

20. Palmer TD, Takahashi J, Gage FH: The adult rat hippocampus contains primordial neural stem cells. Mol Cell Neurosci 8:389-404, 1997

21. Phuphanich S, Scott C, Fischbach AJ, et al: All-trans-retinoic acid: a phase II Radiation Therapy Oncology Group study (RTOG 91-13) in patients with recurrent malignant astrocytoma. J Neurooncol 34:193-200, 1997

22. Raff MC, Abney ER, Miller RH: Two glial cell lineages diverge prenatally in the rat optic nerve. Dev Biol 106:53-60, 1984 
23. Raff MC, Miller RH, Noble M: A glial progenitor cell that develops in vitro into an astrocyte or an oligodendrocyte depending on culture medium. Nature 303:390-396, 1983

24. Rutka JT, De Armond SJ, Giblin J, et al: Effect of retinoids on the proliferation, morphology, and expression of glial fibrillary acid protein of an anaplastic astrocytoma cell line. Int $\mathbf{J}$ Cancer 42:419-427, 1988

25. Savarese A, Annicchiarico-Petruzzelli M, Citro G, et al: Characterisation of a human glioblastoma cell line (LI) expressing hypothalamic and pituitary hormones. Exp Brain Res 89:408-414, 1992

26. Temple S, Raff MC: Clonal analysis of oligodendrocyte development in culture: evidence for a developmental clock that counts cell divisions. Cell 44:773-779, 1986

27. Temple S, Raff MC: Differentiation of a bipotential glial progenitor in single cell microwell. Nature 313:223-225, 1985

28. Warf BC, Fok-Seang J, Miller RH: Evidence for the ventral origin of oligodendrocyte precursors in the rat spinal cord. J Neurosci 11:2477-2488, 1991

29. Wuarin L, Sidell N, deVellis J, et al: Retinoids increase perinatal spinal cord neuronal survival and astroglial differentiation. Int J Dev Neurosci 8:317-326, 1990

Manuscript received March 16, 1998.

Accepted in final form April 2, 1998.

Address reprint requests to: Peter McL. Black, M.D., Ph.D., Division of Neurosurgery, Children's Hospital, 300 Longwood Avenue, Boston, Massachusetts 02115. email: pmblack@bics.bwh.harvard.edu 